\title{
Mycobacterium avium-intracellulare otomastoiditis in a young AIDS patient: case report and review of the literature
}

This article was published in the following Dove Press journal:

HIVIAIDS - Research and Palliative Care

2I February 2013

Number of times this article has been viewed

\author{
J Alexander Viehman ${ }^{1,2}$ \\ Daniel Khalil ${ }^{3}$ \\ Christine Barhoma ${ }^{4}$ \\ Ramy Magdy Hanna' \\ 'Department of Medicine, Olive View- \\ UCLA Medical Center, Los Angeles, \\ ${ }^{2}$ David Geffen School of Medicine \\ at UCLA, Los Angeles, ${ }^{3}$ Department \\ of Biology, University of California, \\ Riverside, ${ }^{4}$ Creighton University \\ School of Pharmacy and Health \\ Professions, CA, USA
}

\begin{abstract}
Mycobacterium avium-intracellulare (MAI) complex is a common opportunistic infection that generally occurs in patients with a CD4 cell count less than 75. Current recommendations for prophylaxis include using a macrolide once a week, while treatment usually requires a multidrug regimen. Disseminated MAI infections often occur in patients who are not compliant with prophylaxis or their highly active antiretroviral therapy (HAART). Many manifestations of MAI infection are well documented in human immunodeficiency virus (HIV) patients, including pulmonary and cutaneous manifestations, but other unusual manifestations such as pericarditis, pleurisy, peritonitis, brain abscess, otitis media, and mastoiditis are sporadically reported in the infectious diseases literature. This case report is of a 22-year-old female who contracted HIV at a young age and who was subsequently noncompliant with HAART, MAI prophylaxis, and prior treatment for disseminated MAI infection. Unsurprisingly, the patient developed recurrent disseminated MAI infection. The patient's presentation was atypical, as she developed severe otomastoiditis and posterior reversible encephalopathy syndrome. The posterior reversible encephalopathy syndrome was thought to be due to the disseminated MAI infection or to immune reconstitution inflammatory syndrome. The infection was confirmed to be secondary to MAI by culture of the mastoid bone. Microbiological analysis of the MAI strain cultured showed resistance to several first-line antibiotics used for prophylaxis against and treatment of MAI. This was likely due to the patient's chronic noncompliance. Otomastoiditis secondary to MAI is extremely rare in adults and has been reported in only four case reports and one case series previously. Improved clinician education in the diagnosis, treatment, and, most important, prevention of MAI and other opportunistic infections is needed. Greater HIV screening, appropriate HAART medication administration, and availability of infectious disease specialists is needed in at-risk populations to help prevent such serious infections. Patient education and greater access to care should serve to prevent medication nonadherence and to enhance affordability of HAART and prophylactic antibiotics.
\end{abstract}

Keywords: opportunistic infection, posterior reversible encephalopathy syndrome, acquired immune deficiency syndrome, macrolide, multidrug regimen, noncompliance

\section{Introduction}

Mycobacterium avium-intracellulare (MAI) is a common opportunistic infection (OI) associated with acquired immune deficiency syndrome (AIDS) patients. The organism is generally ubiquitous in soil and in the environment, but it is readily cleared in most human hosts. ${ }^{1}$ Generally, prophylaxis consists of a once-weekly macrolide in patients with a CD4 cell count less than 75.2,3 Like Mycobacterium tuberculosis infection, MAI infection often necessitates the use of a multidrug regimen, especially in heavily resistant cases. ${ }^{4}$
Correspondence: Ramy Magdy Hanna Department of Medicine, Olive ViewUCLA Medical Center, I4445 Olive View Drive, Sylmar, CA 91342, USA

Email rhannamd8I@yahoo.com 
The most common manifestations of MAI infection are pulmonary and may present with pneumonia-like symptoms or with diffuse lymphadenopathy. ${ }^{1}$ Disseminated MAI infection is common in patients with AIDS. Cutaneous manifestations are less common, but skin lesions have been reported and MAI has been isolated from these skin lesions at times. ${ }^{5,6}$ Less typical presentations include peritoneal, pleural, pericardial, and intracranial presentations as well as osteomyelitis due to MAI infection. ${ }^{1,5-14}$

MAI infections are typically associated with immunosuppressed states, the most common of which is AIDS. One study reported the 5-year all-cause mortality rate of patients with untreated pulmonary MAI infection to be as high as $33 \%$, while the same study found patients with treated MAI infection still had a 5-year mortality rate of $22 \% .{ }^{15}$ In a study from Taiwan, treated disseminated infection was reported as having 30\% mortality. ${ }^{16}$ The underlying immunosuppressed state is likely contributing to these high mortality figures, but it remains evident that MAI infections - particularly, disseminated MAI infections - are a growing menace among AIDS patients and other immunosuppressed patient populations.

The ability of MAI to disseminate in many organs means it is imperative that unusual sites of infection are reported and that the clinician maintains a high level of suspicion and tests for MAI infection in susceptible patients. The present case of otomastoiditis due to MAI represents an unusual site of pathogenesis for this organism. A literature review performed for MAI mastoiditis and otomastoiditis showed only four cases reported in the literature for adult patients, as well as a case series in four children. ${ }^{17-21}$ Another treatment challenge of MAI cases is emerging drug resistance. Drug resistance in MAI infection is being identified as a common reason for treatment failure with macrolides, and treatment alternatives for those who fail macrolide therapy are limited, which is why experts recommend avoidance of the creation of resistant MAI strains. ${ }^{22}$ One Spanish study showed that an alarming $50 \%$ of MAI strains were found to have some resistance to one or more classes of antibiotics used to treat MAI infections (macrolides, quinolones, and rifampin and rifabutin). ${ }^{23}$

The patient in this case report, a 22-year-old female with AIDS and otomastoiditis due to MAI, would only be the ninth patient, and the third adult, reported with this rare manifestation of the OI MAI. In addition to this manifestation, this patient also developed posterior reversible encephalopathy syndrome (PRES) during the course of her MAI treatment; this development was thought to be due to MAI infection or to Immune Reconstitution Inflammatory Syndrome (IRIS).
The patient gave her informed consent for the presentation of this case's findings under the condition of anonymity. Every necessary measure to preserve patient confidentiality has been undertaken in the presentation of this case.

\section{Case report}

A 22-year-old female with a long history of infection with the human immunodeficiency virus (HIV)/AIDS presented to the emergency department at Olive View-UCLA Medical Center with ear pain, weight loss, malaise, and night sweats. The patient was first diagnosed with HIV at age 9, and it was uncertain if she contracted HIV congenitally from her mother, who was HIV positive, or from a subsequent sexual assault by her stepfather, who was also HIV positive. The patient started highly active antiretroviral therapy (HAART) thereafter, but she had been homeless at times and had found it difficult to access care. This difficulty in access resulted in periods of compliance punctuated by periods of medication nonadherence. Prior to this presentation, she had been treated for pulmonary MAI infection as well as Pneumocystis jiroveci pneumonia at an outside institution in 2008 .

In August of 2009 the patient was diagnosed with pulmonary MAI and later with disseminated cutaneous MAI when a rash on her upper arms and thighs was biopsied and the culture returned positive for MAI. In October of 2009 the patient reported a history of a MAI bone infection in her left thumb; subsequently, the infection was surgically removed and replaced with filler, with a plan for a future bone graft. The patient was placed on the following HAART regimen, with which she was noncompliant: emtricitabine-tenofovir, raltegravir, and etravirine. The patient's MAI regimen, with which she was only sporadically compliant, was azithromycin, dapsone, and levofloxacin.

After coming to the emergency department, the patient was started on empiric broad-spectrum antibiotics (vancomycin and piperacillin-tazobactam) and was noted to be hypothermic to $35.5^{\circ} \mathrm{C}$ but hemodynamically stable. The patient's left ear was noted to be tender on manipulation of the pinna, and her left external ear canal was filled with purulent discharge, obscuring her left tympanic membrane from view. The patient's lung and cardiovascular examinations were unrevealing, but hepatomegaly and splenomegaly were noted (liver span, about $20 \mathrm{~cm}$; spleen size, $16 \mathrm{~cm}$ ). Her upper arms and thighs were notable for an annular erythematous lesion that was reportedly biopsied at an outside hospital and showed cutaneous MAI infection. Her neurological examination was most notable for bilateral brisk $(3+/ 5+)$ reflexes. 
The patient's laboratory testing on admission showed that her electrolytes and liver function tests were normal, except for a mildly elevated alkaline phosphatase level and a low serum albumin level, likely secondary to malnutrition. The patient was noted to be leukopenic and her complete blood count showed a microcytic hypochromic anemia, but she was not noted to be thrombocytopenic.

HIV serologies and the HIV polymerase chain reaction test were positive, with HIV RNA at 236,119 copies/ $\mathrm{mL}$. The patient suffered a seizure 2 days after admission and thus cerebrospinal fluid (CSF) analysis was obtained, the results of which were negative. The Veneral Disease Research Laboratory test, M. tuberculosis serological testing, and serology for Coccidioidomycosis immits, Histoplasma Capsulatum, and Cryptococcus Neoformans were all negative.

The patient received a computed tomography (CT) scan of her brain on the day of admission, given the ear and mastoid process pain and the abnormal findings on neurological examination. The CT scan showed opacification of the mastoid air cells and probable left otomastoiditis, so magnetic resonance imaging (MRI) was subsequently performed. A CT of the temporal bone showed left otomastoiditis, generalized cerebral atrophy atypical for age and consistent with HIV, and a rim of aeration around debris in the right mastoid air cells, which also suggested some infection. A sagittal cut of the left mastoid from the preoperative $\mathrm{CT}$, the right mastoid from the preoperative $\mathrm{CT}$, and the post operative $\mathrm{CT}$ scan showing the appearance of the left mastoid process after surgery are shown for comparison in Figure 1.

Based on the patient's weight loss and low CD4 count, a disseminated OI was suspected. Imaging of the ear canal/temporal bone and ear was obtained, given the patient's ear pain. The imaging clearly showed otomastoiditis, and, given the clinical picture of ear pain, a mastoidectomy was scheduled and bone biopsies were obtained to identify the causative organism.

Given the patient's night sweats and weight loss, there was a concern for M. tuberculosis infection - particularly, disseminated tuberculosis. Tuberculosis was not isolated from the bone biopsy tissue culture or from any other body fluid culture. However, MAI was identified on several smears and cultures of induced sputum, an aspirate from a left supraclavicular lymph node biopsy, and several stool specimens. A skin biopsy of the patient's skin lesions again showed MAI on a smear. As mentioned, the patient suffered a seizure 2 days after admission, and CSF was obtained via lumbar puncture as part of a workup to investigate whether her seizure was due to an infectious etiology. The CSF analysis initially
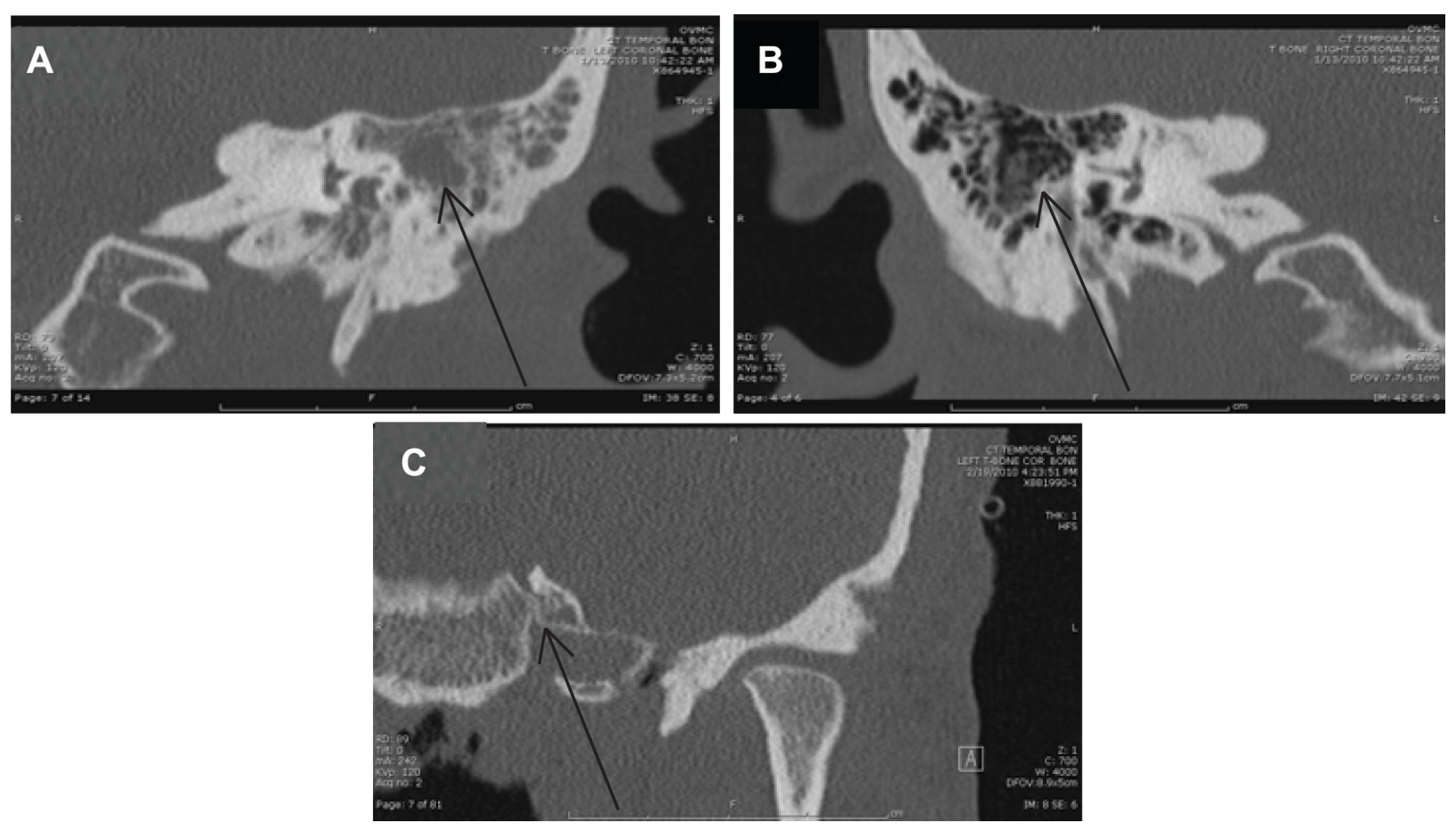

Figure I Imaging of the mastoid processes, demonstrating left otomastoiditis: (A) preoperative temporal bone computed tomography (CT) performed on January I3, 20I0, and showing left-sided otomastoiditis with opacification (arrow indicates inflamed mastoid air cells); (B) preoperative temporal bone CT performed on January I3, 20I0, and showing normal right-sided mastoid air cells without opacification (arrow indicates comparable area that shows the normal appearance of mastoid air cells); (C) postoperative temporal bone CT performed on February 19, 2010, and showing postoperative and postantibiotic resolution of left-sided otomastoiditis (arrow indicates the original area). Note: Some parts of the mastoid process were removed intraoperatively. 
showed no leukocytes, and an acid-fast bacillus smear was also negative, but the culture of the CSF grew MAI several weeks later.

The patient received a brain MRI after the seizure, and another MRI was performed some weeks after that episode. The first brain MRI (Figure 2A) showed left-sided cerebral edema of the occipital and temporal lobes and a radiographic appearance consistent with PRES. The follow-up MRI performed after the patient's discharge from hospital showed resolution of the cerebral edema and the cerebritis/meningitis (Figure 2B).

A chest X-ray of the patient revealed a wide mediastinum and was suggestive of pulmonary hilar lymphadenopathy. A CT scan of the chest was thus performed and this confirmed diffuse hilar lymphadenopathy of the chest. The largest lymph node measured $4.5 \times 3.0 \mathrm{~cm}$. The most demonstrative chest CT images are shown in Figure 3.

\section{Hospital course}

After the diagnosis and the medical and surgical treatment of MAI otomastoiditis, the patient was continued on moxifloxacin, ethambutol, rifabutin, azithromycin, and dapsone. The patient was discharged and readmitted twice with continuing sequelae of disseminated MAI infection. She continues to suffer from overwhelming MAI infection, and she has a low CD4 count that has not yet improved as IRIS has delayed the restart of HAART. The patient has also suffered side effects from the five-medication MAI regimen outlined earlier - namely, pancytopenia due to the disseminated MAI infection and the dapsone. The patient remains on prophylactic trimethoprim-sulfamethoxazole, given her history of Pneumocystis Jirovecii pneumonia; on fluconazole as fungal infection prophylaxis; and on acyclovir as varicella zoster prophylaxis, given her low CD4 count. The patient remains critically ill and her MAI remains refractory to the multidrug treatment regimen.

\section{Discussion}

MAI can be a very challenging OI to diagnose and treat, and it is becoming a greater infectious challenge because of drug resistance. ${ }^{22,23}$ As in the case presented here, patients with MAI - particularly, disseminated MAI - are usually quite immunosuppressed because of transplant medications or advanced HIV/AIDS. ${ }^{11}$ Typically, MAI causes pulmonary symptoms, but diverse disease manifestations including intracranial abscess, bursitis, and many other sites of involvement have been described previously. ${ }^{7,24}$ Like these examples, MAI otomastoiditis is reportable since it is an exceedingly rare manifestation of a fairly common OI. The great variety of unusual presentations is what makes MAI so difficult to treat clinically, and it is important to look for MAI infection in susceptible immunosuppressed patients who may not necessarily present with the usual pulmonary, gastroenterological, and cutaneous symptoms typical of MAI infection.

Otomastoiditis usually requires surgical intervention, and this case was no exception. Bacterial otitis media and otomastoiditis are usually childhood illnesses and are epidemiologically rare in adults. MAI otomastoiditis in an immunosuppressed adult is thus a very noteworthy
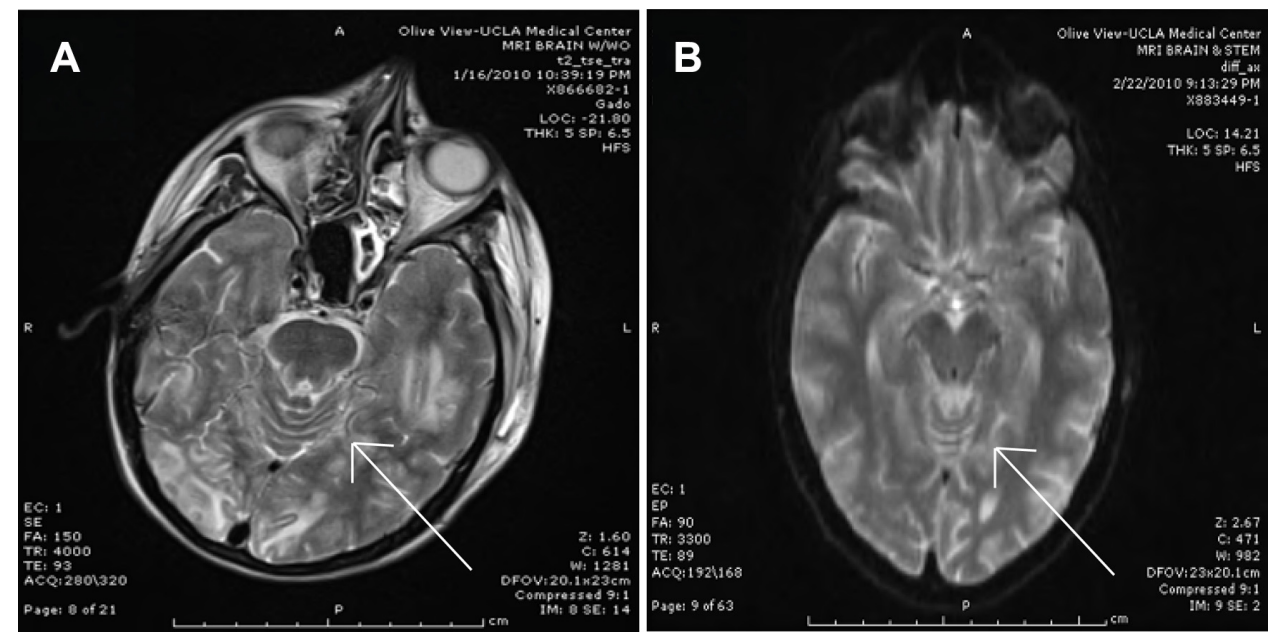

Figure 2 Imaging of the central nervous system post seizure showing posterior reversible encephalopathy syndrome versus Mycobacterium avium-intracellulare cerebritis/ meningitis versus immune reconstitution inflammatory syndrome. (A) Brain magnetic resonance imaging (MRI) with gadolinium performed on January I6, 20I0, and showing bilateral occipital lobe and left temporal lobe edema. The appearance is diagnostic of cerebral edema and could be suggestive of posterior reversible encephalopathy syndrome (arrow indicates gyri with obliterated sulci, suggestive of cerebral edema). (B) Brain MRI with and without gadolinium performed on February 22, 20I0, and showing resolution of the cerebral edema after a long course of treatment for Mycobacterium avium-intracellulare (arrow indicates a comparable area showing gyri with distinct sulci, suggesting resolution of cerebral edema). 

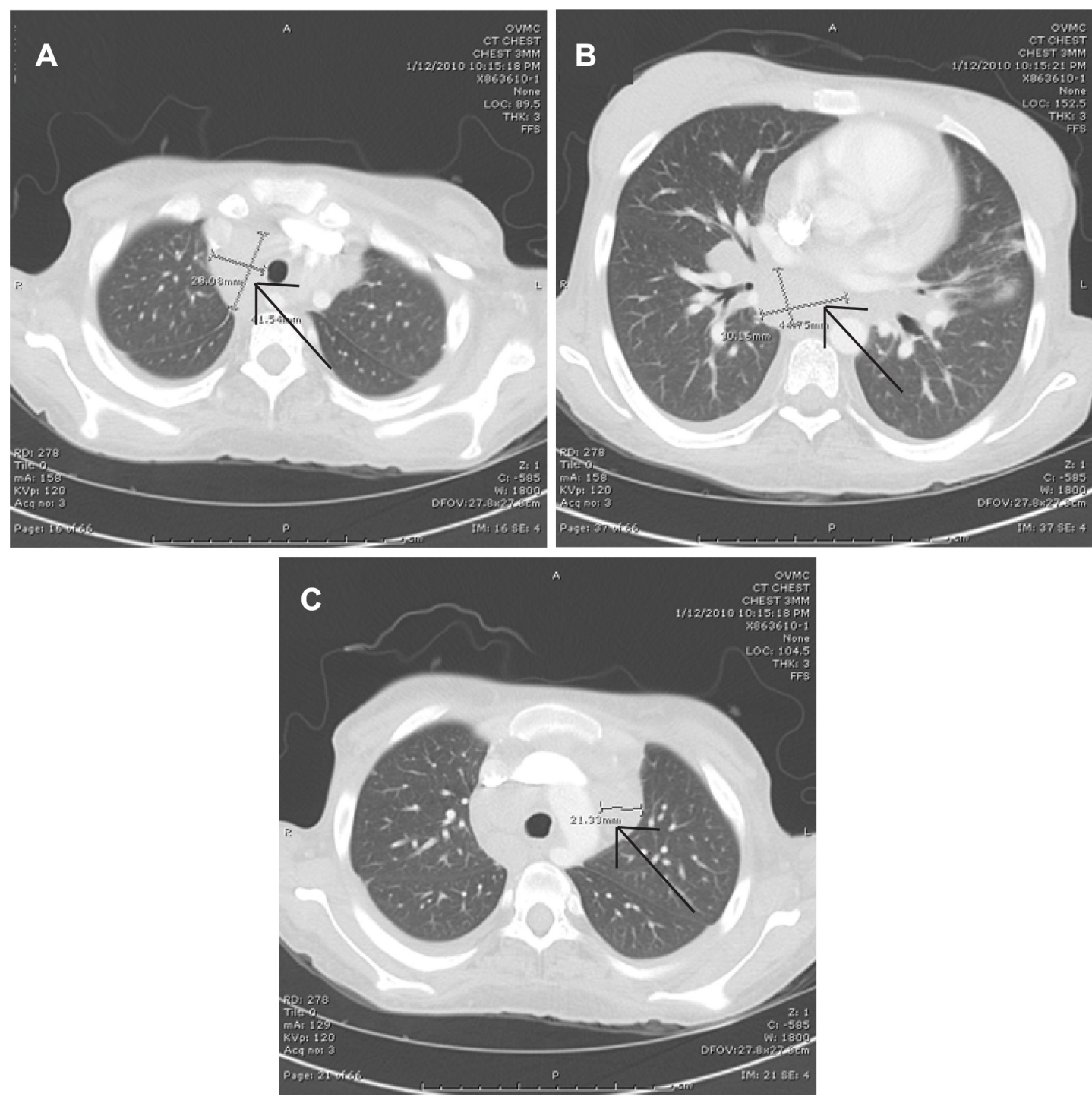

Figure 3 Imaging of the chest showing lymphadenopathy as of January 12, 2010: (A-C) multiple cuts of a computed tomography scan of the chest with intravenous contrast, showing multiple areas of perihilar lymphadenopathy and with the largest lymph node measuring $4.5 \times 3.0 \mathrm{~cm}$ (arrows indicate lymphadenopathy).

presentation, especially since until now it has been reported in only three adults with AIDS. ${ }^{17-21}$ Both the severity of the disseminated MAI in the present patient and the resistance to multiple antibiotics make this case one of great interest. The reported 5-year mortality rates from pulmonary MAI mentioned earlier are rather alarming, but the mortality rate of disseminated MAI is even higher than these..$^{15}$

Another salient feature of this presentation is the patient's development of PRES from either IRIS or central nervous system extension of the MAI. PRES has been previously reported in the setting of MAI infection, but it was attributed to hypercalcemia caused by extensive MAI infection. ${ }^{25}$ In the present case, hypercalcemia was absent and PRES could have been due to MAI extension from the otomastoiditis into the central nervous system, as evidenced by the positive MAI cultures in the CSF, or due to IRIS.

The emphasis in management of MAI infections is on prevention. The high mortality rate, even with pulmonary MAI, demands that attention be paid to prophylactic macrolide administration. Patients with Cavitating MAI lesions have a mortality rate as high as $28 \%$ with treatment, similar to the mortality rate of those patients with disseminated MAI..$^{15,16}$ HIV patients are at a very high risk for mycobacterial infection, with one study showing $55 \%$ of patients diagnosed with a mycobacterial infection (tuberculous or nontuberculous) were HIV positive. ${ }^{16}$ This is quite a problem, as tuberculous and nontuberculous mycobacterium are steadily becoming more resistant, and much of this resistance is occurring in patients susceptible to OIs, such as AIDS patients. ${ }^{16}$ One 
reason for this is that some of these patients are only periodically being compliant with HAART and antimycobacterial therapy because of financial reasons, ultimately resulting in HIV and mycobacterial resistance.

\section{Conclusion}

The emergence of multidrug-resistant tuberculosis and MAI is a hazard to HIV/AIDS patients, immunosuppressed patients, and to the population at large. Improved clinician education in the diagnosis, treatment, and, most important, prevention of MAI and other OIs is needed. Greater HIV screening, appropriate HAART medication administration, and availability of infectious disease specialists is needed in at-risk populations to help prevent such serious infections. Patient education and greater access to care should serve to prevent medication nonadherence and to enhance affordability of HAART and prophylactic antibiotics. Preventing such dramatic immunosuppression by preventing progression from HIV to AIDs will decrease the burden of OIs and will therefore also help decrease the development of drugresistant OIs like the extremely drug-resistant MAI seen in the case reported here.

\section{Disclosure}

The authors report no conflicts of interest in this work.

\section{References}

1. Kasperbauer SH, Daley CL. Diagnosis and treatment of infections due to Mycobacterium avium complex. Semin Respir Crit Care Med. 2008;29(5):569-576.

2. Karakousis PC, Moore RD, Chaisson RE. Mycobacterium avium complex in patients with HIV infection in the era of highly active antiretroviral therapy. The Lancet infectious diseases. 2004;4(9):557-565.

3. Powderly WG. Prophylaxis for opportunistic infections in an era of effective antiretroviral therapy. Clin Infect Dis. 2000;31(2):597-601.

4. Corti M, Palmero D. Mycobacterium avium complex infection in HIV/ AIDS patients. Expert Rev Anti Infect Ther. 2008;6(3):351-363.

5. Bhambri S, Bhambri A, Del Rosso JQ. A typical mycobacterial cutaneous infections. Dermatol Clin. 2009;27(1):63-73.

6. Streit M, Bregenzer T, Heinzer I. Cutaneous infections due to atypical mycobacteria. [Der Hautarzt; Zeitschrift fur Dermatologie, Venerologie, und verwandte Gebiete]. Hautarzt. 2008;59(1):59-70; quiz 1. German.

7. Garrigues GE, Aldridge JM, 3rd, Toth AP, Stout JE. Nontuberculous mycobacterial olecranon bursitis: case reports and literature review. J Should Elbow Surg. 2009;18(2):e1-5.
8. Ishiguro T, Takayanagi N, Saito H, Ubukata M, Yanagisawa T, Sugita Y. Two cases of Mycobacterium avium complex pleuritis. Nihon Kokyuki Gakkai Zasshi .48(2):151-166.

9. Karne SS, Sangle SA, Kiyawat DS, Dharmashale SN, Kadam DB, Bhardwaj RS. Mycobacterium avium-intracellulare brain abscess in HIV-positive patient. Ann Indian Acad Neurol. 2012;15(1):54-55.

10. Montero M, Cercos A, Navarro V, Santos M. Mycobacterium avium intracellulare pericarditis in patients with AIDS. A case report and literature review. Enferm Infecc Microbiol Clin.2007;25(9):606-607.

11. Ramirez J, Mason C, Ali J, Lopez FA. Mycobacterium avium complex pulmonary disease: management options in HIV-negative patients. J La State Med Soc. 2008;160(5):248-254; quiz 54, 93.

12. Shirasaka T. Diagnosis and treatment of tuberculosis or Mycobacterium avium-intracellulare complex infection in HIV-infected patients. Kekkaku. 2007;82(11):845-848.

13. Trupiano JK, Prayson RA. Mycobacterium avium intracellulare otitis media. Ann Diagn Path. 2001;5(6):350-353.

14. Wu UI, Chen MY, Hu RH, et al. Peritonitis due to Mycobacterium avium complex in patients with AIDS: report of five cases and review of the literature. Int J Infect Dis. 2009;13(2):285-290.

15. Ito Y, Hirai T, Maekawa K, et al. Predictors of 5-year mortality in pulmonary Mycobacterium avium-intracellulare complex disease. Int J Tuberc Lung Dis. 2012;16(3):408-414.

16. Chou CH, Chen HY, Chen CY, Huang CT, Lai CC, Hsueh PR. Clinical features and outcomes of disseminated infections caused by nontuberculous mycobacteria in a university hospital in Taiwan, 2004-2008. Scand J Infect Dis. 2011;43(1):8-14.

17. de Juan Martin F, Bouthelier Moreno M, Fernandez Liesa R, Lezcano Carrera MA. Mycobacterium avium otomastoiditis. An Esp Pediatr. 1996;45(6):649-650.

18. Flint D, Mahadevan M, Gunn R, Brown S. Nontuberculous mycobacterial otomastoiditis in children: four cases and a literature review. Int $J$ Pediatr Otorhinolaryngol. 1999;51(2):121-127.

19. Kinsella JP, Grossman M, Black S. Otomastoiditis caused by Mycobacterium avium-intracellulare. Pediatr Infect Dis. 1986;5(6):704-706.

20. Muller B, Kemper J, Hartwig NG, Mooi-Kokenberg EA, van Altena R, Versteegh FG. Mycobacterium avium intracellulare otomastoiditis: case report and literature review. Eur J Clin Microbiol Infect Dis. 2006;25(11):723-727.

21. TerKonda RP, Levine SC, Duvall AJ, 3rd, Giebink GS. Atypical mycobacterial otomastoiditis. Laryngoscope. 1995;105(12 Pt 1):1275-1278.

22. Griffith DE, Aksamit TR. Therapy of refractory nontuberculous mycobacterial lung disease. Curr Opin Infect Dis. 2012;25(2):218-227.

23. Rodriguez JC, Cebrian L, Lopez M, Ruiz M, Royo G. Usefulness of various antibiotics against Mycobacterium avium-intracellulare, measured by their mutant prevention concentration. Int J Antimicrob Agents. 2005;25(3):221-225.

24. Sadek M, Yue FY, Lee EY, et al. Clinical and immunologic features of an atypical intracranial mycobacterium avium complex (MAC) infection compared with those of pulmonary MAC infections. Clin Vaccine Immunol. 2008;15(10):1580-1589.

25. Choudhary M, Rose F. Posterior reversible encephalopathic syndrome due to severe hypercalcemia in AIDS. Scand J Infect Dis. 2005;37(6-7):524-526.
HIV/AIDS - Research and Palliative Care

\section{Publish your work in this journal}

HIV/AIDS - Research and Palliative Care is an international, peerreviewed open-access journal focusing on advances in research in HIV, its clinical progression and management options including antiviral treatment, palliative care and public healthcare policies to control viral spread. The journal welcomes original research, basic science,

\section{Dovepress}

clinical \& epidemiological studies, reviews \& evaluations, expert opinion \& commentary, case reports \& extended reports. The manuscript management system is completely online and includes a very quick and fair peer-review system. Visit http://www.dovepress.com/ testimonials.php to read real quotes from published authors. 